\title{
VACUUM MONITORING SYSTEM OF HLS STORAGE RING
}

\author{
Gongfa Liu, Jingyi Li, Weimin Li, Yong Wang, Caozheng Diao \\ National Synchrotron Radiation Lab., P. O. Box 6022, Hefei, Anhui 230029, P. R. China
}

\begin{abstract}
The vacuum monitoring system of Hefei Light Source (HLS) storage ring is a subsystem of the upgraded HLS control system, which is based upon EPICS. There are 6 Varian vacuum Multi-Gauges controllers around the storage ring of HLS to monitor the pressure of 15 positions. An Industrial PC is used as Input/Output Controller (IOC) and it connects the 6 Varian vacuum Multi-Gauge controllers with serial communication (RS485 mode). The pressure values are displayed in a PC with Linux, which is used as operator interface (OPI). The pressure values are archived in a database and some data analyses are also done.
\end{abstract}

\section{INTRODUCTION}

HLS is a dedicated synchrotron light source in which the electrons circulate at the energy of $800 \mathrm{Mev}$ in the storage ring [1]. The perimeter of the storage ring is 66 meters. There are 6 Varian vacuum Multi-Gauge controllers around the storage ring of HLS to monitor the pressure of 15 positions. The vacuum monitoring system is built on EPICS. An Industrial PC is used as IOC and it connects the 6 Varian vacuum Multi-Gauge controllers via RS485. A tcl/tk program is developed to display the pressure values in histogram. The tcl/tk program resides in a PC with Linux, which is used as OPI. The Self Describing Data Sets (SDDS) file protocol is the basis for a powerful and expanding toolkit of generic programs. The pressure values are archived in a database using SDDS toolkit and some data analyses are also done. [2]

\section{HARDWARE CONFIGUREATIONS}

The vacuum monitoring system of HLS storage ring is built upon EPICS. It is a subsystem of the HLS control system. Fig.1 shows the structure of the vacuum monitoring system.

We use a linux PC with a Pentium III $500 \mathrm{CPU}$ as the OPI. The tcl/tk program runs on it. An IPC with a Pentium II 350 CPU is used as IOC, it communicates with Varian vacuum Multi-Gauge controllers via optically coupled RS485. A RS232 to RS485 card is added to the IPC. In order to provide high-speed communication between OPIs and IOCs, we use 100M Ethernet at the network level. An Intel Pro/100+ Ethernet card was added to the IOC crate to provide an interface between the IOC and the network.

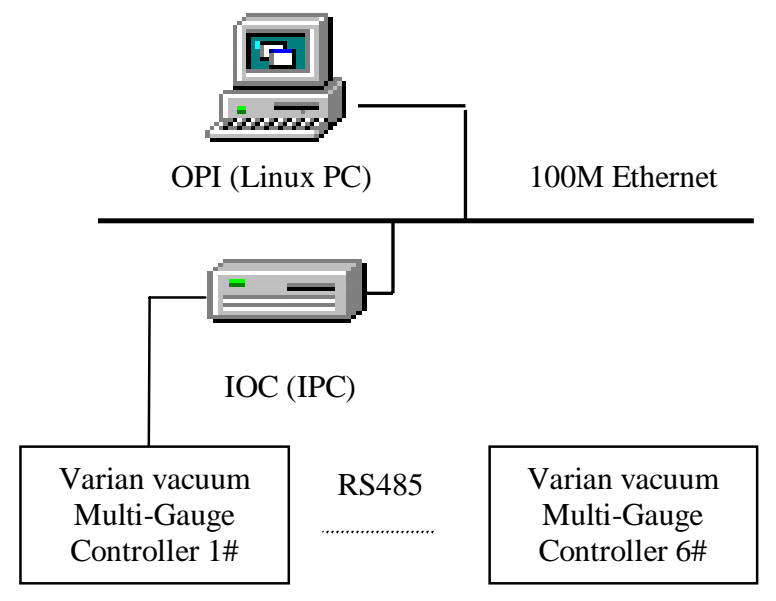

Fig.1 Structure of storage ring vacuum monitoring system.

\section{SOFTWARE OVERVIEW}

\subsection{Software of IOC}

The heart of each IOC is a memory resident database together with various memory resident structures describing the contents of the database. There are about 100 records resident in the IOC. The following record types are mainly used: si (String Input), mbbi (Multi-bit Binary Input), mbbo (Multi-bit Binary Output) and fanout. DCT313 (Database Configuration Tool) is used to create a run time database for the IOC, then the Device Support and Device Drivers (if necessary) are written for each kind of record [3].

Varian vacuum Multi-Gauge provides serial communications (RS485) over the Varian ASCII protocol. The Varian ASCII protocol has its Command/Response format and command set. The user's application must conform to the Varian ASCII protocol. We write a device support and device driver for the Varian ASCII protocol.

In order to protect vacuum Multi-Gauge gauge tube as soon as possible, we add the overpressure protection function in the device support. When pressure equals or exceeds $6 \times 10^{-5}$ pascal, the device support sends the command to all vacuum Multi-Gauge controllers to turn off the gauge tubes. 


\subsection{Software of OPI}

We use a PC with Linux as OPI, a tcl/tk program resides in it. The tcl/tk program is developed to display the pressure values in histogram. Fig.2 is the screen of HLS storage ring vacuum monitoring system. Different color stands for different range of pressure. The blue stands for the average pressure.

The Self Describing Data Sets (SDDS) file protocol is the basis for a powerful and expanding toolkit of generic programs. The SDDS toolkit includes many kinds of tools. We use sddslogger to log the HLS storage ring pressure continuously. The pressure values are archived in SDDS files. Sddsplot is a highly flexible, deviceindependent graphics program. We use sddsplot to show the pressure curve in an optional time range for a position. We can compare the pressure of different time for all 15 positions of the storage ring. Through monitoring the storage ring pressure, we can do some analyses along with the beam intensity and beam lifetime.

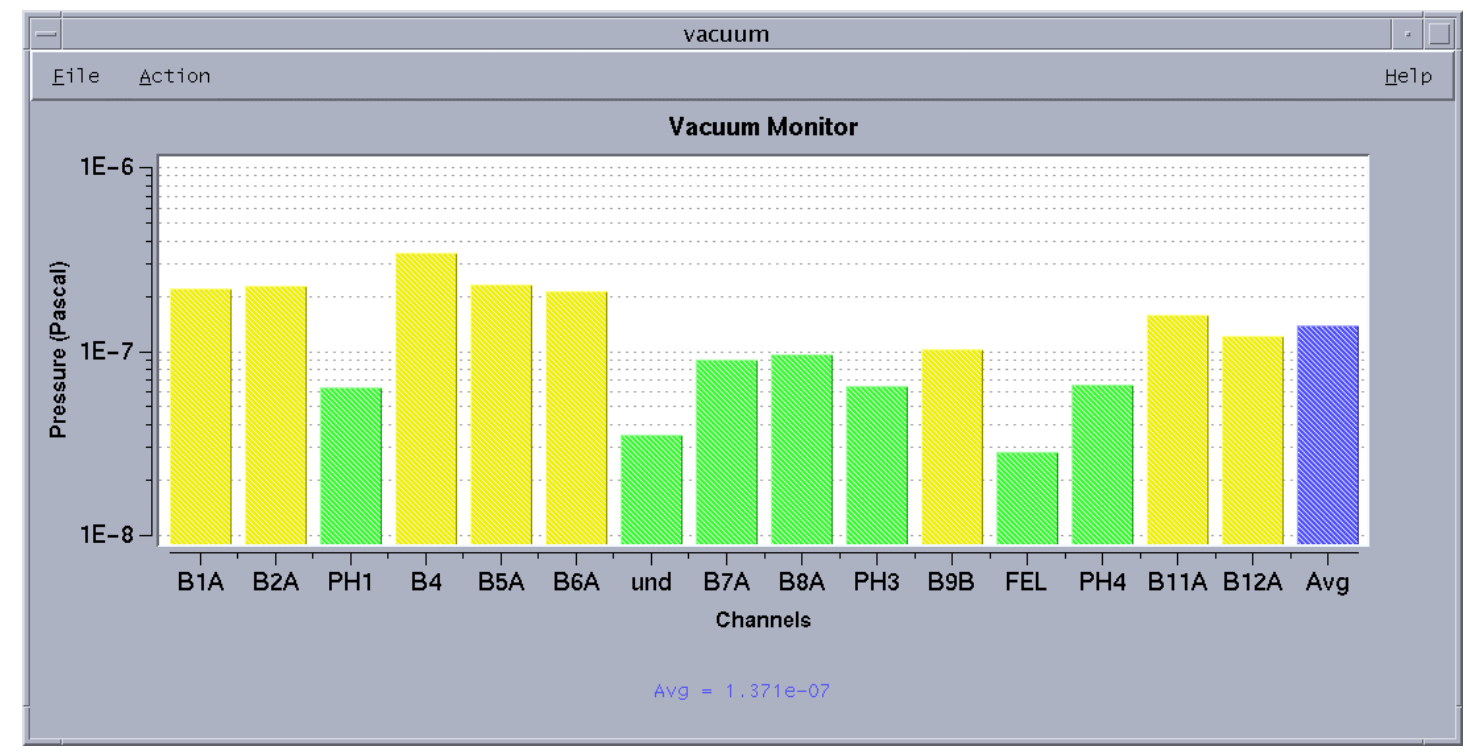

Fig. 2 the screen of HLS storage ring vacuum monitoring system

\section{CONCLUSION}

The vacuum monitoring system of HLS storage ring is a subsystem of the upgraded HLS control system. We have finished the development of the control system of main magnet power supplies. Due to the good extensibility of EPICS, the development for HLS storage ring vacuum monitoring system is easy. The commissioning shows that the monitoring system is reliable because that IPC applies industrial standard and a number of measures are taken to avoid communication error.

\section{REFERENCES}

[1] Weimin $\mathrm{Li}$ et al. "Upgrade Plan for the NSRL Control System". ICALEPCS'97, Beijing, China, 1997.

[2] Michael Borland, "User's Guide for SDDS Toolkit Version 1.15", http://www.aps.anl/sad//asd/oag/.

[3] Martin Kraimer, "EPICS Input / Output Controller (IOC) Application Developer's Guide", EPICS Documentation on http://www.aps.anl.gov/asd/controls/epics/EpicsDocumenta tion. 